\title{
Experiment planning and execution workflow at ASDEX Upgrade
}

\author{
G. Neu, A. Buhler, K. Engelhardt, J. C. Fuchs, O. Gruber, V. Mertens, G. Raupp, J. Schweinzer, \\ W. Treutterer, D. Zasche, T. Zehetbauer and the ASDEX Upgrade Team
}

Max-Planck Institut für Plasmaphysik, Garching, Germany, EURATOM Association

\begin{abstract}
We present the current workflow from experiment proposals to the actual execution and evaluation of discharges at the ASDEX Upgrade tokamak. Requests for experiments are solicited from both within the IPP and from external collaborators in the yearly call-for-proposals, checked for feasibility and compliance with the project's research goals and collected in a proposal database. During the campaign shot requests are derived from the proposals and in weekly operation meetings the requests are mapped to a schedule (shotlist). Before the execution of discharges a complete set of configuration data needs to be assembled. After the execution follows the analysis (including the evaluation of the discharge as to its usefulness for the underlying proposal) and logging of the attained parameters in a physics logbook. The paper describes processes, software tools, and information management showing how they ultimately lead to an improved scientific productivity.
\end{abstract}

Keywords: Experiment campaign, Configuration, Operation, Workflow, Tools

\section{Introduction}

The ASDEX Upgrade project looks back onto nearly 20 years of successful operation. It has recently been mentioned as one of the key experimental devices on the way to a nuclear reactor [1], and has experienced an increasing internationalization, characterized by extensive collaboration with other fusion laboratories and rising numbers of visiting scientists. Besides producing scientific results, one of the main present and future responsibilities is the training of session leaders and experimental fusion scientists for future devices. It is in this context, that we have decided to give a bird's eye view of the organization of experimental campaigns and of the experiences and best practices that have evolved at ASDEX Upgrade.

\section{Campaign preparation}

\subsection{Proposals}

To prepare for a (yearly) campaign (figure1), the ASDEX Upgrade project leader issues an invitation to interested parties, asking for proposals for experiments (or for hardware enhancements, or for diagnostic operation and development). The "call-for-proposals" [2] (a few months before campaign start) sets a frame for the request by listing the task forces (TF) representing broad areas of research interest, major implemented hardware enhancements, technical boundary conditions (such as major plasma parameters), and the planned overall operation schedule. It also describes the criteria for acceptance/prioritization of the proposals and the procedure for preparation of the program .

Proposals are submitted to a proposal database via a web interface. The main informations of experiment proposals are

- a description explaining the scientific rationale and experimental details (such as major plasma parameter ranges and required heating power)

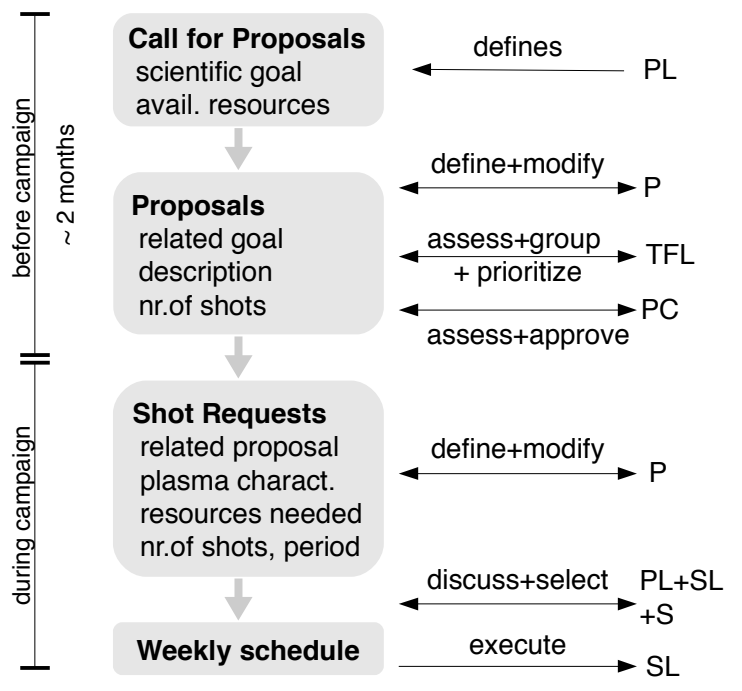

Fig1: Campaign Planning workflow (PC Program Committee, PL Project Leader, TFL Task Force Leaders, SL Session Leaders, P Proponents, S Scientists)

- the number of requested discharges (or shots), classified according to criteria listed in the callfor-proposals

\subsection{Assembling the program}

After the end of the proposal submission phase $(\sim 2$ months after the call) the task force (TF) leaders meet to assess the proposals, grouping them into topics and assigning priorities. Highest priority is given to proposals which exploit new hardware enhancements. The result of the assessment serves as input to a program discussion (ASDEX Upgrade team and guests) where a coherent program for the campaign is prepared. The TF leaders then present the program to the ASDEX Upgrade program committee for final modifications and approval.

Once approved, the complete program for the campaign is available through a wiki on the ASDEX Upgrade web server (restricted access rights). Following 
ASDEX Upgrade's policy of flexibility and user friendliness, post-deadline proposals of interest to the project are accepted.

\section{Campaign execution}

\subsection{Shot requests}

During the campaign, the proponents issue concrete shot requests associated to their proposals by entering them into a shot request database (either directly or via an IPP contact person) using a dedicated web interface. A proposal may lead to several shot requests (e.g. for long-term observations) spread out over the entire campaign. Some important fields of the shot request database entries are

- desired time span for the execution of experiments: not before ... not after ...

- one or more parameter sets describing desired plasma and technical values (modifiable from shot to shot) and the number of requested shots for each parameter set

- machine parameters and diagnostic and control requirements which require longer preparation and are expected to hold for all shots of the request

In weekly physics operation meetings (with remote video participation via H.323) the results of previous experiments and the submitted and still open shot requests are presented and discussed. If accepted, subsequently the latter are compiled into a schedule of discharges (shot list) for execution during the various operation days of that experiment week. Factors such as available resources (plant systems and personnel), backlogs, guest status, upcoming conferences, deadlines for theses and the like are taken into account. Shot requests not related to a proposal are tolerated.

Shot requests are closed automatically when the number of requested useful shots is reached, but may be re-opened by the proponent in case more discharges should be needed. Requests may also be closed manually if further continuation of a proposal is not deemed appropriate.

\subsection{Experimental environment and configuration}

ASDEX Upgrade is equipped with $\approx 50$ diagnostic plant systems evaluating plasma parameters, $\approx 20$ technical plant systems operating the Tokamak's actuators (such as power supplies and magnets, heating and fueling, vessel conditioning, etc.), and a cluster of $\approx 5$ controllers that form the discharge control system (DCS) $[3,4]$

Mapping the desired plasma parameters of a shot request to a valid configuration of plant systems and DCS is far from trivial and the essential part of experiment preparation [5].

Some large fusion experiments choose to define a unique configuration file for a discharge (e.g. JET pulse schedule, W7-X experiment program [6]). ASDEX
Upgrade, however, distinguishes between parts of the configuration which have a long life cycle, such as consistent sets of plant system parameters (created by the plant system's responsible officers), or control parameter sets (e.g. feedback controller gains, computed by control engineers), and the frequently (even from shot to shot) modified discharge programs (DP) - called discharge schedule in [7] - which contain segments with reference waveforms for achieving experimental scenarios.

The feedback control processes [4] of the DCS perform mapping of physics parameters to technical quantities (e.g. density to valve control voltages or plasma cross-section shape to currents of vertical field coils) and special actuator processes of the DCS map unspecific requests (e.g. $5 \mathrm{MW}$ of heating power) to specific actuator systems (e.g. Neutral Beam Injector $\mathrm{x}$ or y), depending on their current availability. This allows for programming scenarios in terms of physics quantities and in technical quantities at sufficient level of abstraction.

By implementing standard ramp-up and eventhandling segments (e.g. for soft-stop) as references or links, DPs will profit from developments (e.g. when major changes of the machine require new breakdown scenarios or when improved event handling strategies are developed). A parameter section of the DP permits to optionally override default control parameters for the scope of a discharge.

To perform its control and monitoring tasks, the application processes (APs) of the DCS need to know about plant settings and control parameter sets. To obtain these, APs query a parameter server which has access to all relevant plant system parameters and to a parameter database holding AP specific parameters (e.g. gain matrices) or functional descriptions of how to compute them. The parameter server also has the task of computing a small set of plant system parameters that directly depend on DP content (e.g. required energy from the flywheel generators, which depends on heating, plasma current and shape)

\subsection{Experiment preparation}

Before a shot can be executed, and preferably before the experiment day, the proponent (or a session leader designated in the proposal) needs to provide an adequate DP. Ideally a reference discharge is mentioned in the shot request, so a template DP can be retrieved from the DP repository [7], to which only minor modification have to be made. If this is not the case, the proponent can use a web interface to search the journal (or physics logbook) database for discharges with similar parameter ranges and scenarios as those of his/her shot request.

The DP editor [7] provides context by allowing to define collections of reference waveforms ("views"), select a reference DP and showing the differences to the current DP, or plot selections of pertinent reference waveforms. An interface to the parameter server is being implemented which will allow to visualize the current state of technical plant systems. Trajectories are mostly 
entered manually but some are imported as results of modeling tools (e.g. from a shape design tool which allows to compute vertical field coil currents for given equilibria, a tool with similar scope is MAXFEA [8]).

A re-implementation of the flight simulator [9] is under way and will be used as a standard tool for DP design by running simulated execution of DPs .

\subsection{Experiment day}

Before the shot-schedule for the day is worked off, test shots for verifying the correct functioning of the safety mechanisms, the peripheral I/O electronics and the magnetic probes are performed. A daily standard $\mathrm{H}-$ mode shot is used as a means to asses the current state of the machine and for documenting its long term behavior.

Due to the complexity of the machine and the importance of avoiding errors and reducing inter-shot time, ASDEX Upgrade operates with two session leaders working together in half-day shifts. One of them assesses the physics of the last discharge and the current plant state and decides on necessary modifications of the actual DP or the further shot-schedule (which will be discussed with the proponent), while the other edits the DPs and updates the journal before the discharge (announcing its principal goals and desired parameters) and after the discharge (entering comments, classifying it, and updating the announced parameters to those actually achieved).

After the session leaders complete the scientific preparation of a discharge they instruct the technical operator to execute it (figure 2). The operator checks readiness of all plant systems, requests validation of the DP against the current state of the plant systems (a parameter server task), and subsequently initiates the configuration of the DCS through the operator interface. Finally the operator starts the discharge. All configuration data is logged under the current shot number. The shot request which is being executed is marked as "started".

The DCS and plant systems execute the shot, record all relevant data, and issue log messages to a log server and the operator interface. A large monitor, visible in the control-room, displays the live image of the inside of the vessel giving an immediate impression on the success of an experiment (occurrence of a plasma, outer shape, visible radiation patterns). Data and log messages are tagged with a 64bit experiment time stamp obtained from the ASDEX Upgrade time system for later comparability of all data.

Between discharges, the daily shot-schedule, a summary of journal database entries for the announced and the already executed discharges, and an overview plot of time traces of important plasma parameters of the last discharge is displayed on the control room monitor (the same information is available on any browser through a customizable web-interface). As soon as the recorded data becomes available - either as raw data or from an automated analysis chain that produces analyzed

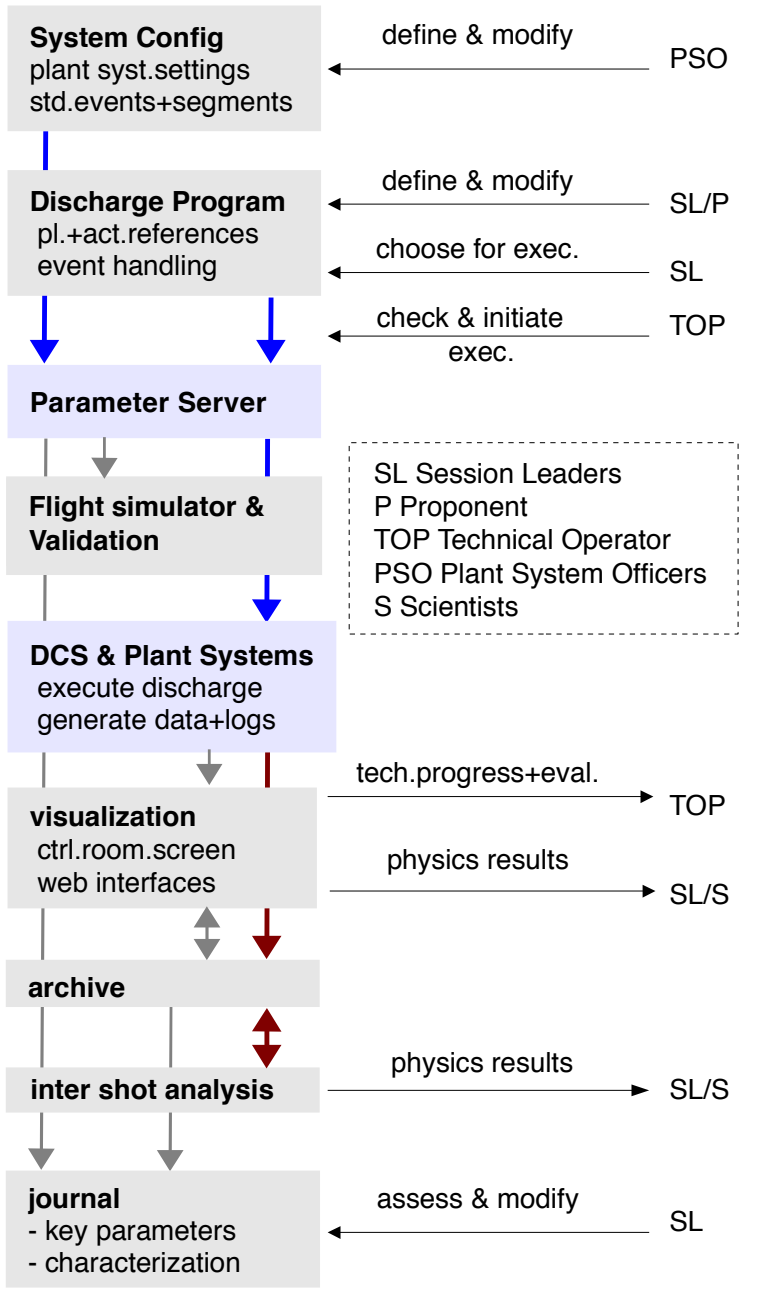

Fig2: Shot execution activities

data for various key parameters - the session leader (sometimes assisted by the proponent) begins with the inter-shot analysis (for an overview of similar inter-shot activities at JET see [10]). He uses a variety of tools for visualization of time traces of plasma and technical parameters (often pre-defined sets for shot-to-shot comparison), 2-D visualization of plasma cross section, or for filtering log messages to assess the pulse and control system behavior. A shot considered useful will be marked as such in the journal database, an information which is also visible in the wiki page of the corresponding proposal (see below).

\subsection{Off-line analysis, remote data access}

Necessary manual interaction in some of the large off-line analysis codes, prevents to perform the complete off-line analysis of ASDEX Upgrade data using analysis chains. A powerful tool, the dependence database (DDB) [11], exists which documents all dependencies between the various diagnostics producing analyzed data (shotfiles [11]). A new run of a modified analysis code which produces a new shotfile edition could use DDB information to trigger computation runs of all codes which directly or indirectly rely on this shotfile. As mentioned above, however, this would require a major restructuring of some higher level diagnostics. 
A web-based tool exists to browse the descriptions of shotfiles, to list all diagnostics for a specific shot, or search for shots with data of specific diagnostics. Other web-tools display timetraces or export (small sets) of them to various formats (excel, e-mail with ascii, etc.).

Remote data access to ASDEX Upgrade data can be achieved by either using the project's MDSplus server or directly accessing the shotfiles in IPP's AFS cell using the shotfile access library

\section{Campaign management tools}

\subsection{The proposals wiki}

The ASDEX Upgrade internal homepage has a link to the proposal wiki, which can be used by proponents and TF leaders to monitor the progress of proposals (useful shots with overview plots), to enter comments, or to navigate to the interfaces of the shot request database (to either enter new requests or modify existing ones) or the journal database (to browse the results of executed shots)

Other functions are available to TF leaders only, e.g. display statistics of proposals and their shot requests or export them into formats (e.g. excel tables) for presentations.

\subsection{The preprint database}

Publications are an important product of experiment campaigns. A pinboard or publication database with a web-frontend allows to enter publications, and to specify or search for entries according to criteria such as type (paper, abstract, other - affects visibility) category (related/not related to ASDEX Upgrade) author(s), date, or status (request for discussion/ submitted/accepted/published).

Scientific coordinators are encouraged to list publications following their proposals on the relevant wiki pages.

\section{Discussion}

The above described workflow is an extremely simplified view of the actual activities revolving around an experimental campaign. It assumes a stable experimental environment and makes no mention of related workflows such as those for assembling plant system configurations, for hardware or software development and commissioning [12], for handling of events such as the unexpected behavior or failures of components, or for plasma theory and computational physics.

Stronger informational integration $[13,14]$ and automation appear to be keys to improve the workflow, but the latter, in particular, collides with the freedom to overrule standard procedures, which is considered to be an important asset by ASDEX Upgrade's scientists to maintain maximum flexibility.

\section{References}

[1] http://ec.europa.eu/research/energy/pdf/978-92-79-10057-

\section{4 en.pdf}

[2] http://www.ipp.mpg.de/ippcms/eng/for/projekte/asdex/organ isation/participation/call for proposal.pdf

[3] G. Raupp, et al., ASDEX Upgrade CODAC overview, Fusion Engineering and Design, Volume 84, Issues 7-11, Proceeding of the 25th Symposium on Fusion Technology (SOFT-25), June 2009, Pages 1575-1579

[4] G. Raupp, G. Neu, W. Treutterer et al., Control process structure of ASDEX Upgrade's new Control and Data Acquisition System, Fusion Engineering and Design, Volume 74, Issues 1-4, Proceedings of the 23rd Symposium of Fusion Technology - SOFT 23, November 2005, Pages 697-705

[5] H. Riemann, et al., Experiment planning using the high level parameter concept, Fusion Engineering and Design, Volume 85, Issues 3-4, Proceedings of the 7th IAEA Technical Meeting on Control, Data Acquisition, and Remote Participation for Fusion Research, July 2010, Pages 478-481

[6] A. Spring, et al., A first W7-X experiment program editor, Fusion Engineering and Design, Volume 85, Issues 3-4, Proceedings of the 7th IAEA Technical Meeting on Control, Data Acquisition, and Remote Participation for Fusion Research, July 2010, Pages 525-528

[7] G. Neu, K. Engelhardt, et al., The ASDEX Upgrade discharge schedule, Fusion Engineering and Design, Volume 82, Issues 5-14, Proceedings of the 24th Symposium on Fusion Technology - SOFT-24, October 2007, Pages 11111116

[8] P. Barabaschi, The MAXFEA Code, Proceedings Plasma Control Technical meeting, ITER JHT, Naka, Japan (1993)

[9] W. Suttrop, L. Höllt, and the ASDEX Upgrade Team: Predictive simulation of tokamak discharge behaviour based on simple scalings.,Proceedings of the 32nd EPS Conference on Plasma Phys. Tarragona, 27 June - 1 July 2005 ECA Vol.29C, P-4.076 (2005)

[10]R. Layne, N. Cook, D. Harting, D.C. McDonald, C. Tidy and JET EFDA contributors, The JET Intershot Analysis: Current infrastructure and future plans, Fusion Engineering and Design, Volume 85, Issues 3-4, Proceedings of the 7th IAEA Technical Meeting on Control, Data Acquisition, and Remote Participation for Fusion Research, July 2010, Pages 403-409

[11]J. Maier, M. G. Pacco Duchs, I. Precht, C. Tichmann, F. Hertweck, Data files and their interdependences: a data base for the tokamak fusion experiment ASDEX Upgrade, Fusion Engineering and Design, Volume 43, Issues 3-4, January 1999, Pages 451-456

[12]A. Kus, et al., Unified software repositories for Wendelstein 7-X: Workflow elements for fusion software development, Fusion Engineering and Design, Volume 83, Issues 2-3, Proceedings of the 6th IAEA Technical Meeting on Control, Data Acquisition, and Remote Participation for Fusion Research, April 2008, Pages 410412

[13]G. Abla, E.N. Kim, D.P. Schissel, S.M. Flanagan, Customizable scientific web portal for fusion research, Fusion Engineering and Design, Volume 85, Issues 3-4, Proceedings of the 7th IAEA Technical Meeting on Control, Data Acquisition, and Remote Participation for Fusion Research, July 2010, Pages 603-607

[14]A. Kramer-Flecken, B. Landgraf, J.G. Krom, Experiment management system--A way towards a transparent Tokamak, Fusion Engineering and Design, Volume 83, Issues 2-3, Proceedings of the 6th IAEA Technical Meeting on Control, Data Acquisition, and Remote Participation for Fusion Research, April 2008, Pages 375-381 